\title{
Numerical solution of problems of plasma dynamics with a neutral layer
}

\author{
Sherzod Khudainazarov ${ }^{1 *}$, Obidjon Karabayev ${ }^{2}$, Ubaydulla Khalmatov ${ }^{3}$, and Akhrorbek \\ Numonov ${ }^{1}$ \\ ${ }^{1}$ Department of Theoretical and Structural Mechanics, Tashkent Institute of Irrigation and Agricultural \\ Mechanization Engineers, 100000 Tashkent, Uzbekistan \\ ${ }^{2}$ Tashkent Islamic Institute named after Imam Bukhari, Tashkent, Uzbekistan \\ ${ }^{3}$ Tashkent State Technical University after I.Karimov, Tashkent, Uzbekistan
}

\begin{abstract}
Mathematical modeling on a computer is widely used in problems of magnetic hydrodynamics and dynamics of ionized gas (plasma). In these problems, in addition to the equations of mass, momentum, and energy balance that are common in continuum mechanics, it is necessary to solve Maxwell's equations for the electromagnetic field. An urgent and interesting problem in the theory of ionized gas is the problem of changing the topology of the magnetic field in plasma during the processes of magnetic field lines reconnection. Such situations are characteristic of plasma processes in the Earth's magnetosphere (natural processes and the ones resulting from active experiments in space) and in laboratory facilities for the creation of closed configurations. Because of the difficulties of diagnostics, mathematical numerical modeling is of great importance. The article presents twodimensional mathematical models of the processes of magnetic field lines reconnection occurring in plasma with finite conductivity in the vicinity of the neutral surface. Effective numerical algorithms are constructed for solving problems of plasma dynamics in the approximation of one-fluid magnetohydrodynamics. Calculations are conducted taking into account dissipative processes. The problem of transportation and interaction of closed plasma configurations in a single computational domain was solved.
\end{abstract}

\section{Introduction}

Mathematical modeling as a scientific direction is still quite young, and its active development continues. The main features of modern mathematical modeling are related to the fact that in the last decade it is rapidly losing its "academic" features of a purely scientific and narrowly professional field. Along with the classical problems, numerous problems that arise in the practical use of methods and simulation results are currently being solved.

The methods of mathematical modeling are briefly expressed by the well-known "model algorithm - program" triad, formulated by Academician A.A. Samarsky [1,2] - one of the "founding fathers" of mathematical modeling. These methods received their development in

${ }^{*}$ Corresponding author: scherzodshox77@,mail.ru 
the form of the "computational experiment" technology developed by A. A. Samarsky's school and designed to study the phenomena of the surrounding world when a full-scale experiment turns out to be too expensive and complicated. A computational experiment precedes a full-scale one. Full-scale experiments were "ordered" based on the results of mathematical modeling. A few years later, in three physics laboratories of the Russian Federation, the T-layer was reliably registered almost simultaneously on different experimental setups; after that, the operation principle of the MHD generator with a T-layer became finally clear to technologists and engineers.

Currently, plasma with its nonlinear properties has become one of the most important objects of mathematical modeling and computational experiment. The processes occurring in plasma in an electromagnetic field at ultrahigh temperatures are objects of interest from both scientific and practical points of view. Experimental studies are conducted in many research laboratories worldwide, including large generators Angara-5-1 and S-300 in Russia, the MAGPIE generator in the UK and the world's largest generator " $Z$ " in the USA. Plasma and its nonlinear properties are being widely studied at present $[5,9]$.

The tempting prospect of solving the energy problem of humanity can be associated with controlled thermonuclear fusion (CTF), the study of which is conducted, in particular, by a computational experiment. Plasma generators of new generations are now being used for experimental studies of CTF. Specific types of such generators are the plasma current circuit breaker installations and the installations to study the Z-pinch behavior. For a comprehensive study of plasma dynamics on such installations, a full-scale experiment is not enough since it does not give a complete and detailed picture of what is happening; the design features of the diagnostic parts allow measuring the plasma characteristics only at certain discrete points of time.

One of the promising directions in the creation of high-intensity pulsed sources of soft X-ray radiation for the CTF program with inertial plasma confinement, and the study of the properties of materials under conditions of the destructive effect of absorbed radiation on them, is based on pulsed generators of the mega-ampere range, for which the load is an imploding Z-pinch with characteristic dynamics times of the order of several tens of nanoseconds. This makes it possible to obtain pulses of soft X-ray radiation with the power of hundreds of terawatts [3]. For this, the rapid compression of thin-walled cylindrical shells (liners) is used. Experiments on the collapse of liners are conducted in several laboratories worldwide, including at the largest generators in Russia - Angara-5-1 [4] and S-300 [6, 7, 8], in Britain - the MAGPIE generator [5] and in the USA - the world's largest generator "Z" [9]. In recent decades, high energy density physics has been developing rapidly. The impact of strong shock waves on the substance, intense fluxes of laser radiation, electron and ion beams, processes in various pinch devices and magnetocumulative generators, collision of rapidly flying bodies - this is not a complete list of examples of energy concentration in dense media.

Practical applications are associated with modern research in the field of energy, inertial thermonuclear fusion, and space technology. A study of the dynamics of a plasma flow switch in the conduction mode within the framework of a two-fluid MHD model with allowance for the Hall effect was conducted in [10]. Criteria were obtained for the operating modes of the flow switch with a predominance of the MHD, Hall and mixed-type effects. The penetration of a magnetic field along the cathode due to the Hall effect is described. The influence of a combination of the Hall effect and MHD effects on the formation and evolution of a low-density region in the near-electrode space is shown.

In [11], the results of numerical calculations of the formation of cluster targets for various experimental conditions are presented. It was shown that the shape of the nozzle used could have an extremely strong effect on the spatial distribution of clusters. The parameters of argon and krypton cluster targets are compared under the same conditions of their formation. 
The study in [12], describes the development of mathematical models of polydisperse flows and the creation of basic software for the numerical implementation of these models in an automated system for modeling practically important cases of flows in geometrically complex computational domains.

In [13] the results of the development of difference schemes with positive approximation on unstructured grids for equations of hyperbolic, elliptic, and parabolic types in complex domains of integration and with a complex structure of numerical solution are presented. The paper presents the results of the study on the development of a system designed to manage complex computational problems and facilitate their creation, such as the problems with many complex interacting areas of complex geometry and rheology. A new object model was proposed that has sufficient generality for constructing a variety of dynamic computing environments operating both on a single computer and on a network. The results of testing the proposed methods and the results of numerical studies conducted using these methods are presented in relation to some problems of continuum mechanics and are of independent scientific interest. The implementation of an experiment to study the possibility of using the method of joint numerical solution of the equations of a one-fluid two-temperature MHD model and a kinetic model describing the dynamics of states of ionization of a plasma substance is described. Good agreement with the results of physical experiments on pulsed plasma generators is shown. The use of methods of parallel computations made it possible to significantly accelerate the calculations of experimental options.

Thus, the practical use of plasma configurations with a closed magnetic field undoubtedly requires a deep analysis of all stages and aspects of the process: formation, axial compression, stability, transportation, and heating. From the above, it follows that the study of the processes of magnetic field lines reconnection in plasma is of great interest both for understanding complex astrophysical phenomena and for the use in laboratory and practical applications.

The study of the dynamics of neutral current sheets in plasma has the main goal of elucidating the possibility and efficiency of converting the magnetic energy of the current in the current sheet into the kinetic energy of the directed motion of charged particles [13]. Investigation of the possibility of accelerating particles in a current sheet involves several stages, one of which is obtaining a stable current sheet.

\section{Formulation of the problem}

At the initial point in time $\mathrm{t}=0$, the plasma is at rest $(\mathrm{v}=0)$ in a cylindrical chamber of radius $\mathrm{R}$ and length $L$ with a magnetic field vanishing on the surface of radius $r_{0}$. In the considered case of an axially symmetric flow $\partial / \partial \varphi=0$, we have a poloidal magnetic field $H=\left(H_{r}, 0, H_{z}\right)$ and a toroidal current $\mathrm{j}=\left(0, \mathrm{j}_{\varphi}, 0\right)$.

At the first stage of calculations, i.e. when forming a closed configuration, the solution of the system is considered for a rectangular domain $0 \leq \mathrm{r} \leq 1,0 \leq \mathrm{z} \leq \mathrm{L}$ [17]. At the initial time, the density has a maximum at $r=r_{0}$, i.e. on a zero surface of the magnetic field, and is given in the following form:

$$
\rho(r, z, 0)=1+c h^{-2} \alpha\left(r-r_{0}\right)
$$

Coefficient $\alpha$ determines the width of the neutral layer. Gas-dynamic pressure satisfies the condition of plasma equilibrium in a magnetic field:

It has a maximum value at $\mathrm{r}=\boldsymbol{r}_{\mathbf{0}}$,

$$
P+\frac{H^{2}}{8 \pi}=\text { const }
$$

$$
P(r, z, 0)=P_{0}-t h^{2} \alpha\left(r-r_{0}\right)
$$

here $\mathrm{P}_{0}$ is the value of gas-dynamic pressure on a zero surface magnetic field $\mathrm{r}=\mathrm{r}_{0}$. Initial distribution of magnetic field strength is: 


$$
H_{z}(r, z, 0)=t h \alpha\left(r-r_{0}\right), H_{r}(r, z, 0)=0,
$$

The distribution of gas-dynamic pressure and density is used to determine temperature:

$$
\mathbf{T}(\mathbf{r}, \mathbf{z}, \mathbf{0})=\mathbf{P}(\mathbf{r}, \mathbf{z}, \mathbf{0}) / \boldsymbol{\rho}(\mathbf{r}, \mathbf{z}, \mathbf{0})
$$

Magnetic field potential A $(r, z, 0)$ is determined by numerical integration by Simpson's method:

$$
A(r, z, 0)=(1 / r) \int_{0}^{r} r H_{z} d r
$$

The boundary conditions for the problem under consideration are as follows:

$$
\begin{aligned}
& \text { for } \mathrm{r}=0, \mathrm{u}=\mathrm{A}=0, \frac{\partial f}{\partial r}=0, \text { where } \quad \mathrm{f}=\{\mathrm{v}, \mathrm{P}, \rho\}, \\
& \text { for } \mathrm{r}=1, \partial \rho / \partial \mathrm{r}=\partial \mathrm{P} / \partial \mathrm{r}=0, \\
& \mathrm{u}=\mathrm{v}=0, \mathrm{~A}=\mathrm{A}_{0}+\mathrm{A}_{1} \exp \left(-\mathrm{z}^{2} / \mathrm{l}^{2}\right)[1-\exp (-\omega \mathrm{t})],
\end{aligned}
$$

The planes $\mathrm{z}=0, \mathrm{z}=\mathrm{L}$ are the planes of symmetry with obvious boundary conditions:

$$
\frac{\partial f_{1}}{\partial r}=0, \text { where } f_{1}=\{u, P, \rho, A\} \quad \text { for } z=0, z=L
$$

The calculations were performed for $\alpha=30 ; \mathrm{r}_{0}=0.35 ; 1=0.8 ; \mathrm{A}_{1}=0.5 ; \omega=0.5 ; \mathrm{P}_{0}=1.1$; $\mathrm{L}=4.5$.

The process of forming closed configurations is considered with account for the conductivity and viscosity of plasma caused by collective processes. In the experiments, the reconnection of field lines is performed using key turns, located at the end of the chamber. In the calculations, the key turns are modeled by a time increment of vector potential at the boundary. An increment $r=1$ leads to a disturbance of the balance and plasma motion to the $r=0$ axis. The presence of plasma finite conductivity $(\nu \neq 0)$ leads to the rupture and reconnection of initially antiparallel magnetic field lines with the formation of a closed configuration - a compact torus.

The initial equilibrium state of plasma in the absence of external disturbance $\left(A_{1}=0, \eta=0\right.$, $v=0)$ is kept with a high degree of accuracy. This testifies to the good properties of numerical algorithms. To study the reconnection process, it is necessary to introduce quantitative characteristics in order to use them in analyzing the plasma flow. One of the quantitative characteristics of the reconnection process is the difference of magnetic fluxes in sections $\mathrm{z}=0$ and $\mathrm{z}=\mathrm{L}$, for any point in time $\mathrm{t}$. It is defined as:

where

$$
Q(\mathrm{t})=\left(Q_{\mathrm{L}}-Q_{0}\right) / Q_{\mathrm{L}}
$$

$$
\begin{aligned}
& Q_{L}=2 \pi r_{1} \mathrm{~A}\left(r_{1}, \mathrm{~L}, \mathrm{t}\right), \\
& Q_{0}=2 \pi r_{0} \mathrm{~A}\left(r_{0}, \mathrm{~L}, \mathrm{t}\right),
\end{aligned}
$$

Here $r_{1}, r_{0}$ are the coordinates of the minimum values of $A r$ at the boundaries of the domain.

\section{Results and Discussion}

With an increase in the coefficient of magnetic viscosity $v$, the function $Q(t)$ which determines the degree of reconnection increases. The reliability of these considerations is confirmed by the calculations. When a closed configuration is formed, along with reconnection, the magnetic flux dissipation also occurs. It equals to:

where

$$
\mathrm{D}=\left(\mathrm{D}_{\mathrm{L}}-\mathrm{D}_{0}\right) / \mathrm{D}_{0}
$$

$$
D_{L}=2 \pi r_{1} A\left(r_{1}, L, t_{1}\right), D_{0}=2 \pi r_{0} A\left(r_{0}, L, 0\right),
$$

The calculation results showed that large values of the amplitude and frequency of disturbances lead to the rapid formation of a closed configuration.

Let us now consider the process of transportation and merging of asymmetric closed configurations. As mentioned above, after the formation and longitudinal compression of closed configurations, it is possible to study the process of transportation and interaction along the z-axis. 


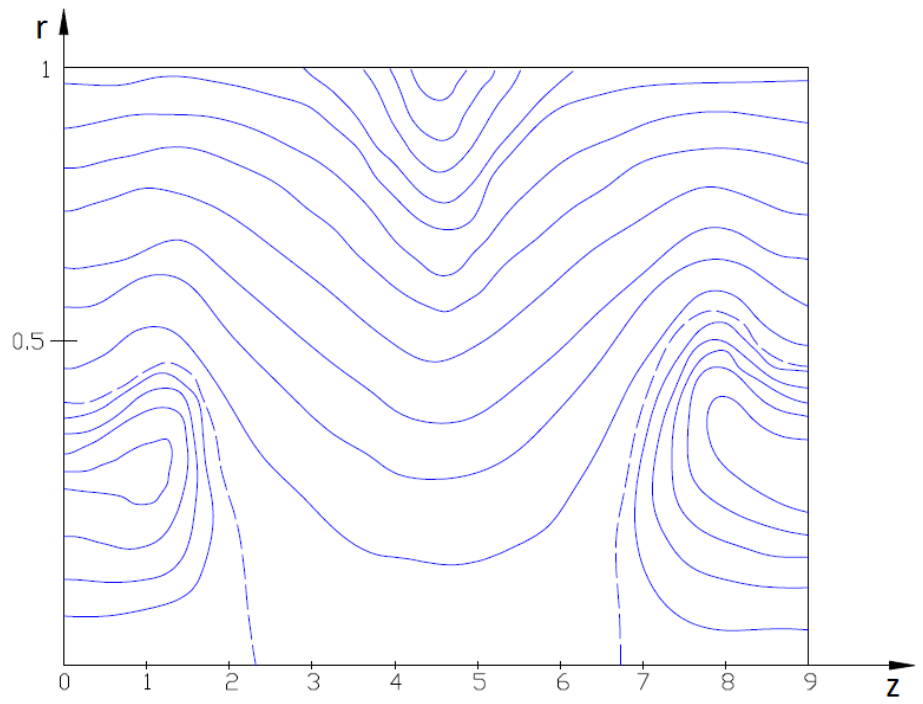

Fig. 1. Topology of the magnetic field at time $t=6.25: r$ is the radius of the tube; $z$ is the length of the tube; ----- - is the separatrix line, where the magnetic field strength is $\mathrm{H}=0$; - isolines, where the magnetic field strength $\mathrm{H}>0$

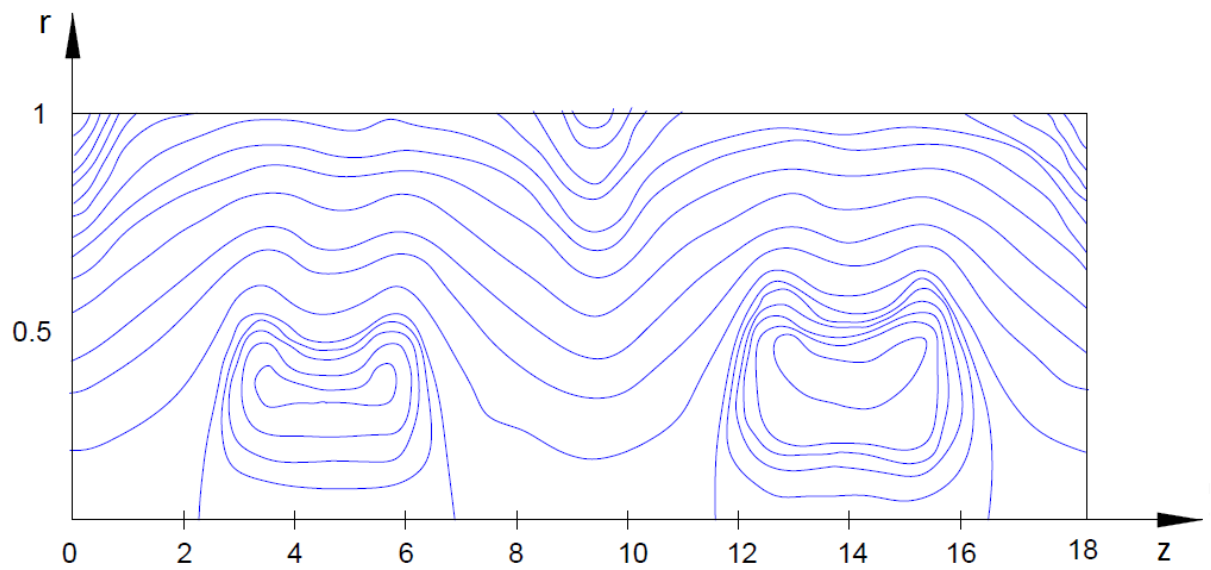

Fig. 2. Isolines of the magnetic field at time $t=6.25$ for the computational domain $0 \leq z \leq 4 L: r$ is the radius of the tube; $z$ is the length of the tube

We will assume that the solution obtained at $\mathrm{t}=6.25$ serves as the initial data for the interaction problem. The topology of the magnetic field of the tori at $\mathrm{t}=6.25$ is shown in Figure 1, where the solution domain is $0 \leq \mathrm{r} \leq 1,0 \leq \mathrm{z} \leq 2 \mathrm{~L}$.

We transform the solution to the problem for $\mathrm{t}=6.25$; in the segment $0 \leq \mathrm{z} \leq 2 \mathrm{~L}$ symmetrically with respect to the plane $\mathrm{z}=0$ and $\mathrm{z}=2 \mathrm{~L}$. Then we get a new computational domain $0 \leq \mathrm{z} \leq 4 \mathrm{~L}$, with the following topology of the magnetic field (Fig. 2).

The calculated times $\mathrm{t}=4.25$ and $\mathrm{t}=6.25$ are special in the study of reconnection processes. Therefore, in addition to graphs, it is necessary to add tables for more detailed coverage of the processes under consideration. Time $t=4.25$ refers to the complete reconnection of the magnetic field lines; and $\mathrm{t}=6.25$ to the beginning of the interaction process, see Table 1 : 
Table 1. Change in the average characteristics of a compact torus at a moment in time $\mathrm{t}=$ 4.25

\begin{tabular}{cccc}
\hline $\begin{array}{c}\text { Average } \\
\text { characteristics }\end{array}$ & $\begin{array}{c}\text { Time } \\
\mathrm{t}=4.25\end{array}$ & $\begin{array}{c}\text { Left torus } \\
\mathrm{t}=6.25\end{array}$ & $\begin{array}{c}\text { Right torus } \\
\mathrm{t}=6,25\end{array}$ \\
\hline $\mathrm{W}_{\mathrm{T}}$ & 4.559 & 3.439 & 4.649 \\
$\mathrm{~W}_{\mathrm{H}}$ & 0.273 & 0.187 & 0.667 \\
$\mathrm{~W}_{\mathrm{k}}$ & 0.030 & 0.020 & 0.167 \\
$\mathrm{~W}$ & 4.862 & 3.645 & 5.483 \\
$\mathrm{~T}$ & 0.645 & 0.642 & 0.548 \\
$\mathrm{P}$ & 1.798 & 1.803 & 1.941 \\
$\mathrm{M}$ & 4.910 & 3.686 & 5.669 \\
$\mathrm{~V}$ & 2.731 & 2.045 & 2.920 \\
\hline
\end{tabular}

As seen from Table 1 , at $\mathrm{t}=4.25$ both tori are identical. During this time, the temperature remains almost unchanged. The average characteristics of closed configurations are determined as follows:

$$
\begin{aligned}
& \mathrm{W}_{\mathrm{T}}=2 \pi \iint \frac{\mathrm{rP}}{\gamma-1} \mathrm{drdz} \text { - thermal energy, } \\
& \mathrm{W}_{\mathrm{H}}=2 \pi \iint \mathrm{r} \mathrm{H}^{2} \mathrm{drdz} \text { - magnetic energy, } \\
& \mathrm{W}_{\mathrm{H}}=2 \pi \iint \mathrm{r} \mathrm{u}^{2} \mathrm{dr} \mathrm{dz} \quad \text { - kinetic energy, } \\
& \mathrm{W}=\mathrm{W}_{\mathrm{T}}+\mathrm{W}_{\mathrm{H}}+\mathrm{W}_{\mathrm{k}} \quad \text { - total energy, } \\
& \mathrm{T}=\mathrm{W}_{\mathrm{T}}(\gamma-1) / \mathrm{V} \quad \text { - average plasma temperature, } \\
& \mathrm{m}=2 \pi \iint \mathrm{r} \mathrm{dr} \mathrm{dz} \quad \text { - mass of plasma, } \\
& \mathrm{V}=2 \pi \iint \mathrm{rdr} \mathrm{dz} \quad \text { - volume. }
\end{aligned}
$$

The average characteristics of the left and right closed configurations before and after merging are of interest. They are shown in Table 2:

Table 2. Average characteristics of a compact torus at subsequent times

\begin{tabular}{cccccc}
$\begin{array}{c}\text { Average } \\
\text { characteristics }\end{array}$ & $\begin{array}{c}\text { Left torus } \\
\mathrm{t}=17.3\end{array}$ & $\begin{array}{l}\text { Right } \\
\text { torus }\end{array}$ & Left torus & $\begin{array}{l}\text { Right } \\
\text { torus } \\
\mathrm{t}=24.7\end{array}$ & $\mathrm{t}=29.1$ \\
$\mathrm{t}=24.7$ & & & \\
\hline $\mathrm{V}$ & 1.486 & 2.113 & 1.116 & 1.912 & 2.313 \\
$\mathrm{~W}_{\mathrm{H}}$ & 0.109 & 0.304 & 0.084 & 0.288 & 0.327 \\
$\mathrm{~W}_{\mathrm{T}}$ & 3.352 & 4.412 & 2.789 & 4.249 & 5.675 \\
$\mathrm{~W}_{\mathrm{k}}$ & 0.009 & 0.023 & 0.023 & 0.056 & 0.053 \\
$\mathrm{P}$ & 2.360 & 2.245 & 2.598 & 2.460 & 2.547 \\
$\mathrm{M}$ & 3.507 & 4.744 & 2.960 & 4.516 & 6.168 \\
\hline
\end{tabular}

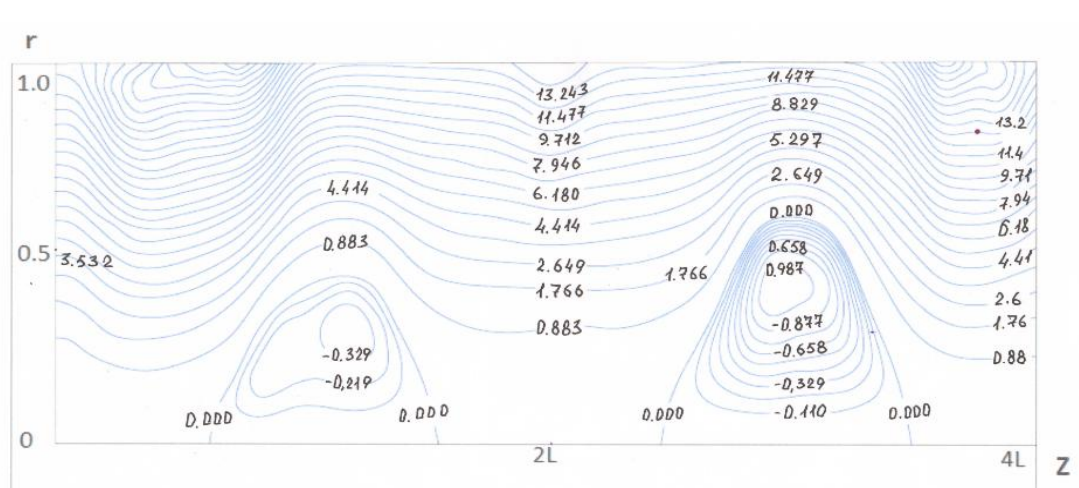

Figure 3. Topology of the magnetic field at time $t=15.7: r$ is the radius of the tube; $z$ is the length of the tube 


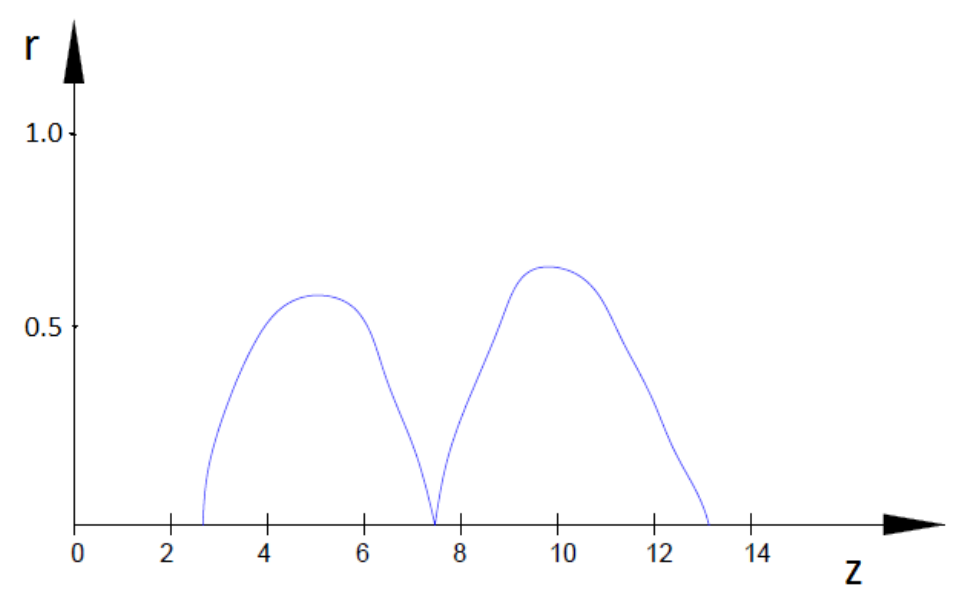

Fig. 4. Topology of the magnetic field at the beginning of the merger: $r$ is the radius of the tube; $z$ is the length of the tube

The definition of average characteristics is given above. During transportation and interaction, closed configurations change their shape (Fig. 3-5). During transportation, the weight is reduced by 1.6 times. The plasma temperature remains constant. During the interaction, the magnetic energy drops by a factor of 2 .

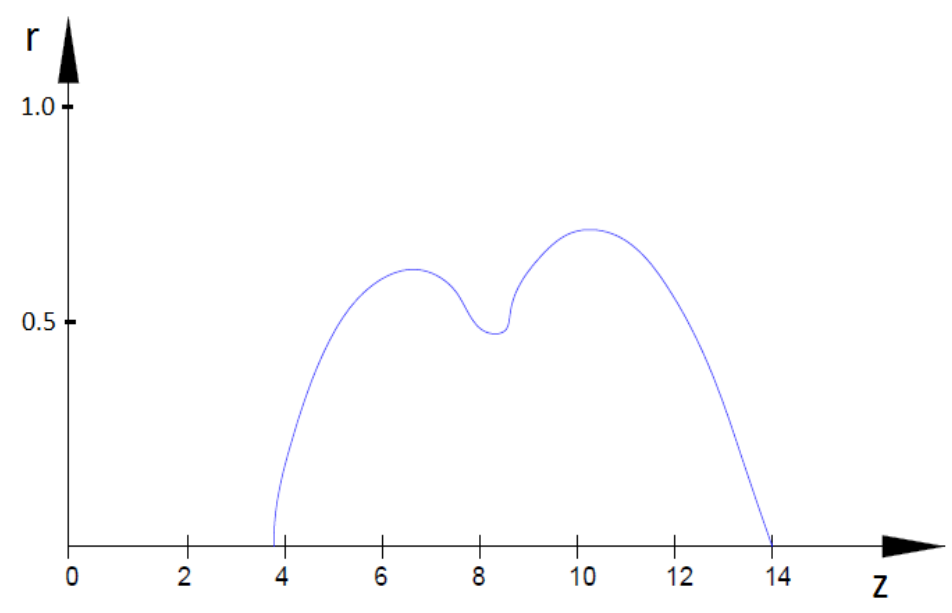

Fig. 5. Magnetic field topology after fusion: $r$ is the radius of the tube; $z$ is the length of the tube

The results of calculations allow us to conclude that the merging of closed configurations leads to the possibility of increasing the energy and lifetime with the formation of a general structure of the magnetic field.

\section{Conclusions}

Based on the results of the studies, the following conclusions can be drawn:

1. Algorithms for the numerical solution of problems of reconnection processes in the approximation of one-fluid magnetohydrodynamics with a modification of finite-difference schemes were developed (an explicit scheme with account for the sign of velocity in terms 
containing transfer, a scheme with an implicit approximation of viscous terms, a two-step Lax-Wendroff scheme, an implicit splitting scheme);

2. It was shown that the process of interaction and merging of closed plasma configurations has a pulsating character;

3. It was shown that the merging of closed plasma configurations leads to the formation of a general structure of the magnetic field with the possibility of increasing its energy and time; 4. The unsteady two-dimensional problem of plasma dynamics in complex configurations with a neutral layer, accompanied by a change in the topology of the magnetic field, was solved.

\section{References}

1. A.A. Samarskii, New York - Basel, Marcel Dekker 761 (2001)

2. A.A. Samarskii, A.P. Mikhailov, London and New York, Taylor and Francis (2002)

3. Yu.G. Kalinin, Mathematical modeling 11, 63-80 (2000)

4. L.E. Aranchuk, Yu.G. Kalinin, A.S. Kingsep, V.A. Skoryupin, V.V. Yan'kov, JETP 44(5), 970 (1976)

5. S.V. Lebedev, Rev. Sci. Instrum 72, 671 (2001)

6. A.A. Akunets, S.S. Anan'ev, Yu.L. Bakshaev, I.V. Volobuyev, A.A. Zelenin, European Physical Journal D 54(2), 499-502 (2009)

7. D.Kh. Morozov, E.O. Baronova, I.U. Senichenkov, Plasma Physics Reports 33, 906 (2007)

8. L. Jakubowski, M. Sadowski , E. Baronova, Chech Journal of Plasma Physics 54, 1-6 (2004)

9. J.D. Huba, NRL Plasma Formulary, Naval Research Laboratory, Washington DC: 68 (2013)

10. V.P. Zhukov, Plasma Physics 45(1), 156-169 (2005)

11. V.P. Zhukov Plasma Physics 31(8), 43-49 (2005)

12. V.P. Zhukov, G.I. Dudnikova, A.G. Frank, Computational Technologies 9(3) 39-49 (2004)

13. N.G. Basov Proc. FIAN USSR 74, 168 (1974)

14. Yu.A. Berezin, G.I. Dudnikova, Nauka, Moscow (1985)

15. W.T. Armstrong, R.K. Linford, Phys. Fluids 24(11), 2068-2089 (1981)

16. A.G. Eskov, V.K. Korshunov, 3826/7 Moscow 40 (1983)

17. G.I. Dudnikova, V.P. Zhukov, O.I. Karabaev, Problems of the dynamics of a viscous fluid 139-143 (1985)

18. O.I. Karabaev, Reports of the Academy of Sciences of Uzbekistan, Science 2, 16-17 (1987)

19. G.I. Dudnikova, O.I. Karabaev, Reports of the Academy of Sciences of Uzbekistan, Science 5, 14-15 (1990)

20. V.A. Vshivkov, G.I. Dudnikova, Publishing house of the Institute of Theoretical and Applied Mechanics, Siberian Branch of the USSR Academy of Sciences, Siberia (1983)

21. H.E. Petschek, Proc. AAS - NASA Sympos. Physics, Solar Flfres, Washington (1964)

22. E.R. Priest, Solar Physics 86, 33-45 (1983) 